\title{
Butterfly wings exhibit spatial variation in chromatin accessibility
}

\section{Heidi Connahs*, Mainak das Gupta and Antónia Monteiro*}

Affiliation:

Department of Biological Sciences, National University of Singapore

*Corresponding authors:

Heidi Connahs, Antónia Monteiro

Email:

12 hconnahs@gmail.com, antonia.monterio@nus.edu.sg

Keywords: FAIRE-seq, Chromatin accessibility, Butterfly wings, Morphology, GC content, Hox genes, cofactors

\section{Abstract}

18 Butterfly wings exhibit a diversity of patterns which can vary between forewings and hindwings and spatially across the same wing. Regulation of morphological variation involves changes in how genes are expressed across different spatial scales which is driven by chromatin dynamics during development. How patterns of chromatin dynamics correspond to morphological variation remains unclear. Here we compared the chromatin landscape between forewings and hindwings and also across the proximal and distal regions of the hindwings in two butterfly species, Bicyclus anynana and Danaus plexippus. We found that the chromatin profile varied significantly between the different wing regions, however, there was no clear correspondence between the chromatin profile and the wing patterns. In some cases, wing regions with different phenotypes shared the same chromatin profile whereas those with a similar phenotype had a different profile. We also

28 found that in the forewing, open chromatin regions (OCRs) were AT rich whereas those in the

29 hindwing were GC rich. GC content of the OCRs also varied between the proximal and hindwing

30 regions. These differences in GC content were also reflected in the transcription factor binding 31 motifs that were differentially enriched between the wings and wing regions. Our results suggest

32 that distinct wing patterns may result from the interaction of pioneer factors, including Hox genes,

33 differentially opening chromatin in different wings and wing regions and cooperating with other

34 transcriptions factors, that show preferences for specific GC content, to function either as activator

35 or repressors of nearby genes. 


\section{Introduction}

37 Butterflies are renowned for their striking wing patterns which can vary dramatically between forewings and hindwings and across different regions and surfaces of the wing. Numerous studies have characterized spatial patterns of gene expression across different regions of the wing (Ferguson and Jiggins 2009; Ferguson et al. 2011; Prakash and Monteiro 2018; Hanly et al. 2019; Banerjee and Monteiro 2020) and also identified genes that regulate specific colors and pattern elements (Martin and Reed, 2010; Reed et al., 2011; Kunte et al., 2014; Mazo-vargas et al., 2017;

43 Matsuoka and Monteiro, 2018; Connahs et al., 2019). The spatial and temporal expression pattern

44 of any gene is defined by chromatin dynamics in which specific regions of chromatin start to 45 become open during development, via the binding of pioneer factors, facilitating the binding of 46 further transcription factors to open chromatin regions (OCRs), which ultimately drive patterns of gene expression (Shlyueva et al. 2014). But precisely how these changes in chromatin dynamics translate to morphological variation is still poorly understood.

Most studies to date, which have examined the chromatin landscape of whole tissues, generally show that at the genome-wide level, chromatin accessibility is surprisingly invariant. Studies in the butterflies Junonia and Heliconius, have shown similar chromatin profiles between forewings and hindwings despite them displaying different color patterns (Lewis and Reed 2018; Burg et al. 2019; Lewis et al. 2019). In Drosophila, a comparison of the chromatin landscape across different appendages also revealed that the same enhancers were accessible at most genomic regions with the exception of the loci around master regulators (McKay and Lieb, 2014). Similar to the studies in Junonia and Heliconius, changes in the chromatin profile corresponded with different stages of development, whereas at the same developmental stage even tissues with very different phenotypes had a remarkably similar profile.

Based on these findings how can we explain the development of different morphologies if the

62 chromatin landscape is largely identical? McKay and Leib suggested that master regulators, which

63 were the few genes they found to be differentially accessible as well as expressed between different 64 appendages in flies (e.g. Ultrabithorax $(U b x)$ and vestigial), are responsible for the morphological 65 differences. These genes would bind to the same set of accessible enhancers of downstream genes effecting changes in gene expression across appendages. Differential chromatin accessibility for 
67 two key regulators was also found in Junonia and Heliconius butterflies (Lewis and Reed 2018;

68 Burg et al. 2019; Lewis et al. 2019), where the only difference observed in the chromatin profile

69 of forewings and hindwings was around the loci of $U b x$, which defines hindwing identity

70 (Weatherbee et al. 1999; Tomoyasu 2017; Matsuoka and Monteiro 2021) and Abd-a (Heliconius

71 only) a neighboring gene to $U b x$ (Deutsch 2005). Other key genes such as optix, previously shown

72 to regulate red wing pattern elements in Heliconius, did not have distinct chromatin profiles

73 surrounding its OCRs between forewings and hindwings even when only one wing had a red

74 pattern element (Lewis. et al. 2019). A ChIP-seq also showed that Optix protein itself binds the

75 same OCRs in both wings, and deletion of OCRs containing optix binding sites leads to disruption

76 of these patterns (Lewis et al. 2019). How information processed at those OCRs contribute to the

77 differential expression of optix in forewings and hindwings remains, thus, unclear.

An alternative explanation to McKay and Leib's suggestion is that different wing phenotypes do

80 in fact correspond to changes in the chromatin profile but that capturing the chromatin profile at

81 the whole tissue level is insufficient for detecting spatial differences occurring at a finer resolution.

82 A study examining the chromatin profile across different regions of a Drosophila embryo showed

83 distinct spatial differences in the chromatin landscape when compared to the profile obtained using

84 whole embryos (Bozek et al. 2019). Examining the whole embryo resulted in an averaging out of

85 the chromatin signal thus masking spatial differences in chromatin accessibility. These findings

86 indicate that previous studies, using whole butterfly wings, or whole fly appendages, may have

87 missed spatial differences in the chromatin landscape that could be associated with differences in

88 wing/appendage phenotypes.

90 Here we examined whether the wing chromatin landscape corresponds to differences in wing color

91 patterns. In the African butterfly, Bicyclus anynana, both forewings and hindwings display

92 eyespots on the distal margin of the wings whereas the proximal wing region is largely

93 homogeneous with few distinct pattern elements. We hypothesized that the chromatin profile

94 would differ between the distal and proximal wing tissue, but would be more similar between the

95 distal regions of the forewing and hindwing which have eyespots. We also compared the chromatin

96 profile of the proximal and distal regions of the Monarch butterfly, Danaus plexippus which

97 exhibits monochromatic spots along the distal margins of both wings and different patterning in 
more proximal wing regions. We then investigated whether these two butterflies shared regions of open chromatin that were highly conserved at the sequence level, and whether these regions exhibited functional conservation, i.e., were open in the same wings and/or wing regions compared to non-conserved OCRs. Finally, we performed a motif discovery analysis to examine whether the regions of open chromatin in different wings or wing regions were enriched in particular transcription factor binding motifs. This analysis aimed to discover transcription factors important

104 in regulating differences in wing color pattern phenotypes.

\section{Results}

\section{Open chromatin profile varies between different wings and wing regions}

108 We first annotated the FAIRE-seq data using peaks observed in IGV, highlighting the significant peaks called from MACS2 to check whether open chromatin regions (OCRs) were open across all

110 wing regions regardless of phenotype as observed in Heliconius and Junonia. For this analysis we

111 focused on the significant peaks around 6 genes known to be involved in wing patterning (Distal112 less, Ecdysone receptor (EcR), spalt, decapentaplegic (dpp), wingless and engrailed). For $B$.

113 anynana, we compared the open chromatin landscape between the distal regions of the forewing 114 (FWD) and hindwing (HWD) and also between the proximal and distal regions of the hindwings 115 (HWP and HWD). For D. plexippus we compared the proximal and distal regions of the hindwings.

116 In contrast to $B$. anynana, the data for the forewing (FW) represents the entire wing rather than 117 just the distal region (Fig 1). For both butterfly species, we found that the chromatin landscape 118 varied significantly between forewings and hindwings and between the proximal and distal regions 119 of the hindwing (Figs 2-3, S1). We next examined the number of shared OCRs between the 120 different wings and wing regions. For this analysis we used the FAIRE-seq peaks extracted from 121 all scaffolds. For D. plexippus, 41,608 significant peaks were observed across the whole genome $122(\mathrm{FW}=21178, \mathrm{HWD}=8911, \mathrm{HWP}=11519)$. For $B$. anynana, we had data for 31 scaffolds and 123 observed 629 significant peaks $(\mathrm{FWD}=240, \mathrm{HWD}=272, \mathrm{HWP}=117)$. We found that in both 124 species the majority of overlapping OCRs were observed between the HWP and FWD/FW regions 125 which have very different wing phenotypes (Fig 4). In D. plexippus the fewest overlaps were 126 observed between the FW and HWD regions. In B. anynana, we did not observe any peaks that 127 were uniquely shared between the FWD and HWD regions despite the presence of eyespots in 128 both of these wing regions (Fig 4). 


\section{Comparison of the chromatin landscape between $B$. anynana and $D$. plexippus}

130 The two butterfly species have very different wing patterns and therefore we would expect to

131 observe a different chromatin landscape even around the same genes. However, it is possible that

132 some OCRs may be specific to particular wing regions and perhaps functionally conserved

133 between both butterfly species. We first compared the chromatin profile around six genes known

134 to be involved in wing patterning. In B. anynana, we observed 19 regions of open chromatin

135 across the $231 \mathrm{~kb}$ scaffold containing the Distal-less gene. Twelve of these peaks were found in

136 the HWD only, four regions were open only in the FWD, one was open only in the HWP, and

137 one open region was shared between the FWD and HWP region at the TSS (Fig 2). In contrast,

138 in the same region around the Distal-less gene in D. plexippus, there was a single region of open

139 chromatin in the HWD, however at the TSS there was also an overlapping peak between the FW

140 and HWP region and another peak overlapping the HWD and HWP regions. Thus, in both

141 butterflies there is a shared peak in the proximal hindwing and forewing tissue at the TSS.

142 Comparing the open chromatin profiles around the remaining 5 genes, the profiles are largely

143 different, however for EcR (Fig 2), spalt and $\operatorname{dpp}$ (Fig 3) the TSS of these genes overlaps with

144 open chromatin in the HWD and HWP regions for both butterfly species. Chromatin profiles for

145 wingless and engrailed are shown in Fig. S1.

Most conserved peaks are open in different wing regions in the two butterfly species

148 Using mVista for genome alignments, we compared the scaffolds between these two butterfly

149 species to identify regions of sequence conservation with a minimum threshold of $50 \%$. We then

150 examined whether any of these regions of sequence conservation overlapped regions of open

151 chromatin, to look for conserved peaks between the two butterflies. In the region around Distal-

152 less we identified 5 peaks which shared sequence conservation $>50 \%$ between the two

153 butterflies. In all 5 cases the conserved peaks were open in different wing regions in each

154 butterfly. Interestingly, peak 2 which overlaps the TSS in both FWD and HWP libraries is not

155 conserved with the region that overlaps the TSS in the FW and HWP libraries in D. plexippus but

156 rather it shares sequence conservation with a different a peak in the HWD and HWP libraries.

157 However, for EcR and spalt (Figs 2 and 3), the peaks at the TSS (peaks 5 and 2 respectively) are

158 conserved and open in the same wing regions in both butterflies. For EcR and $d p p$ (Figs 2 and 3),

159 peaks 2 and 3 respectively, are also open in forewing tissue in both butterflies. 


\section{Motif enrichment in regions of open chromatin}

161 We next examined whether there was any evidence of motif enrichment in regions of open

162 chromatin between the different wing regions. For D. plexippus we first pooled the HWD and

163 HWP data allowing us to compare the FW and the HW. MEME-chip identified 50 motifs which

164 were enriched in the FW versus the HW in D. plexippus (E-value: 1.2e-1458) (Fig S2A). The top

165 hit matched to known motifs for the transcription factors, Jigr1 (Jing Interacting Gene

166 Regulatory) and Bab1 (Bric-a-Brac). Significant secondary motifs were also identified using

167 SpaMo (E-value: 1.58e-11). MEME-chip identified 47 motifs that were significantly enriched in

168 the HW versus the FW (E-value: 7.0e-2424), with the top hit showing similarity to the

169 transcription factors Mad (Mothers against dpp) and Crol (Crooked legs) (Fig S2B). Significant

170 secondary motifs were also identified using SpaMo (E-value: 1.12e-13) which resemble the

171 primary motif. Comparing OCRs across the hindwing, between HWD versus the HWP, MEME-

172 chip identified 55 GC-rich motifs enriched in the HWD region with the top hit matching to

173 Escargot and Trithorax-like (GAGA factor, GAF) (E-value: 5.8e-1652) (Fig S2C). The CentriMo

174 feature in MEME-chip identified this motif as having a central distribution within the OCRs.

175 Significant secondary motifs were also identified using SpaMo (E-value: 2.53e-8) which were

176 also GC-rich. For the comparison between the HWP versus the HWD regions, 37 motifs were

177 enriched in the HWP (E-value: 3.3e-564), an AT-rich motif matching Bab1 was identified as the

178 top hit (Fig S2D). Significant secondary motifs were also identified using SpaMo which are also

179 AT-rich.

180

181 For B. anynana, 10 motifs were identified as enriched in FWD versus HWD wings (E-value:

182 1.3e-003), 6 motifs were significantly enriched in HWD versus FWD (E-value: 8.8e-009) and 3

183 motifs were significantly enriched in HWD versus HWP (E-value: 4.0e-007). For each of these

184 comparisons the top hits had no similarity to any known motif. For HWP versus HWD, 4 motifs

185 were significantly enriched (E-value: 5.1e-004) with the top hit matching to the transcription

186 factors Mad, Enhancer of split $\mathrm{m} \beta$, helix-loop-helix and odd paired.

\section{GC content of motifs and OCRs varies between different wings/regions}

189 Based on the MEME-chip analysis, we observed that motifs enriched between different wings and 190 regions appeared to differ in GC content. To examine whether there was a statistical difference in 
191 GC content we calculated the percent GC content for each motif for each wing/region in $D$.

192 plexippus where we had the most data. We found that HW motifs had a significantly higher GC 193 content than FW motifs $\left(\log _{\mathrm{e}}\left(\mathrm{W}_{\text {Mann-Whitney }}\right)=3.87, \mathrm{r}=-0.96, \mathrm{p}<0.0001\right)$ (Fig 5A). We next looked 194 to see if the GC bias for the HW motifs was explained by differences in GC content in the OCRs 195 themselves. We found that HW OCRs had a significantly higher GC content than the FW OCRs $196\left(\log _{\mathrm{e}}\left(\mathrm{W}_{\text {Mann-Whitney }}\right)=18.26, \mathrm{r}=-0.61, \mathrm{p}<0.0001\right)($ Fig 5B). The overall GC content of the genome 197 is $28 \%$, and the GC content of the FW OCRs is slightly lower at $24 \%$. Both are much lower than 198 the HW OCRs at 39\% which is more similar to the coding regions at $46 \%$. To examine whether 199 the GC content is consistent across the HW itself, we repeated these analyses for the HWD and 200 HWP OCRs. The motifs for the HWD OCRs had a significantly higher GC content than for the 201 HWP OCRs $\left(\log _{\mathrm{e}}(\mathrm{W}\right.$ Mann-Whitney $)=7.53$, r=-0.87, p<0.0001) (Fig 5C) and likewise the GC content 202 of the HWD OCRs was significantly higher than the HWP OCRs ( $\log _{\mathrm{e}}\left(\mathrm{W}_{\text {Mann-Whitney }}\right)=18.22$, $\mathrm{r}=-$ 203 0.6, p<0.0001) (Fig 5D). Together these results show that HWD OCRs are enriched in GC content, 204 whereas the HWP and FW OCRs display a much lower GC content which is reflected in the AT205 enriched motifs. We next examined whether the higher GC content in the HWD region could be 206 due to these OCRs being longer. We found that the average length of the OCRs was significantly 207 different between all groups, however the FW had the longest OCRs and the HWP region had the 208 shortest OCRs $(\mathrm{KW}=419.7, \mathrm{r}=-0.01, \mathrm{p}<0.0001)($ Fig S3A).

We next looked to see if this pattern held in B. anynana comparing the FWD with the HWD data. 211 OCRs were extracted from all 31 scaffolds. Only a small number of motifs were found in the 212 MEME-Chip analysis. The motifs enriched in the FWD OCRs had a GC content of 19\% $(n=13)$ 213 compared to the motifs enriched in the HWD OCRs which had a much higher GC content of $69 \%$ 214 ( $n=14)$. We also compared the GC content of the FWD and HWD OCRs and found that the GC 215 content was significantly higher in the HWD $\left(\log _{\mathrm{e}}\left(\mathrm{W}_{\text {Mann-Whitney }}\right)=9.47, \mathrm{r}=-=0.46, \mathrm{p}<0.0001\right)(\mathrm{Fig}$ 216 S4A). The combined scaffolds had a GC content of $32 \%$ compared to the whole genome which 217 was $15 \%$ (5630 scaffolds). The GC content of the coding regions was much higher at $51 \%$.

219 We also compared the OCRs between the HWD versus HWP regions. This analysis produced a 220 small number of motifs, however in this comparison the HWP motifs (n=9) had a much higher GC 221 content than the HWD motifs $(n=6)$ at $86 \%$ versus $27 \%$ respectively. Comparing the GC content 
222 of the OCRs for these scaffolds, we observed a significantly higher GC content for the HWP than

223 the HWD $(\mathrm{t}=-4.53, \mathrm{df}=230.64, \mathrm{p}<0.0001)($ Fig S4B). The length of the OCRs were also

224 significantly different for each wing region with the longest OCRs found in the HWD region and 225 the shortest in the HWP region $(\mathrm{KW}=30.71, \mathrm{r}=-0.06, \mathrm{p}<0.0001)(\mathrm{Fig} \mathrm{S} 3 \mathrm{~B})$.

227 Comparison of the GC content in open chromatin overlapping genomic elements

228 Finally, we examined whether the GC content varied in regions of open chromatin that

229 overlapped different genomic elements to determine whether the differences in GC content were

230 explained by OCRs open in specific parts of the genome (Fig S5-7). Focusing on the data from

231 D. plexippus, we found that for each wing/region most of the open chromatin mapped to

232 intergenic and intronic regions, and that the GC content showed significant differences between

233 various genomic elements within each wing/wing region. While these differences were small,

234 more variation was observed for the GC content in HWP OCRs that overlapped exons, the

235 promoter and the TSS (Fig S6). Overall, we found that the GC content was, on average, similar

236 across all genomic elements within each wing region, with a higher average in the distal

237 hindwing.

\section{Discussion}

240 We examined whether the chromatin landscape varies with wing pattern variation in two species 241 of butterflies which exhibit some similarities and differences between forewings and hindwings

242 and spatially across the hindwings. We hypothesized that in wing regions with similar color

243 patterns such as the distal tissue with eyespots in B. anynana, the forewings and hindwings might

244 share a similar chromatin profile. This hypothesis is based on previous work showing that the

245 development of eyespots in forewings and hindwings is generally regulated by the same set of

246 genes (Monteiro et al. 2015; Özsu et al. 2017; Connahs et al. 2019; Banerjee et al. 2020).

247 Although the forewings and hindwings differ in the number of eyespots, we predicted that we

248 might detect a common signature in the chromatin profile surrounding eyespot-associated genes

249 at 24 hours post-pupation. Our data however did not support this hypothesis. We did not

250 identify any OCRs that were shared in both wing tissues with eyespots around the loci of 6 genes

251 known to be important in eyespot development, nor were any overlaps found for the remaining

25225 scaffolds. As we did not have FAIRE-seq data for the entire genome, we cannot rule out the 
253 possibility of shared OCRs between the distal forewing and hindwings in other regions of the 254 genome.

Interestingly, although $D$. plexippus has a very different wing pattern with simple white spots 257 along the distal margins of both wings, we found that that the fewest overlapping OCRs were observed between the distal hindwing and forewing tissue. As we profiled chromatin in the entire forewing we will have missed spatial differences in this wing, therefore the number of shared OCRs between the distal forewing and distal hindwing might be even smaller. Taken together our results do not show any evidence that similar phenotypes on different wing tissues share a similar chromatin profile at the same stage of development. Nor did our findings support those in Junonia and Heliconius where the chromatin profile was shown to be similar in forewings and hindwings at the same developmental stage regardless of phenotype (Lewis and Reed 2018a; Burg et al. 2019; Lewis, Geltman, Pollak, Rondem, and Belleghem 2019). Our findings suggest that there are distinct differences in how the chromatin is regulated between forewings and

Although we observed regional differences between the proximal and distal regions of the hindwing which differ morphologically for both butterflies, we also found OCRs that are shared across the entire hindwing including at the TSS. This could be due to the same genes being expressed across the entire wing tissue albeit at different levels in the proximal and distal

273 regions. An unexpected finding was that most OCRs were shared between the distal forewing

274 and proximal hindwing regions in B. anynana which show no morphological similarity.

275 Similarly, we also found that the highest number of overlapping OCRs were between the

276 forewing and proximal hindwing regions in D. plexippus. This observation is difficult to

277 understand with our current data, and we later discuss some potential scenarios that may explain

278 these patterns. Overall our results appear to be more in line with those from Bozek et al., (2019)

279 who observed regional differences in open chromatin across the Drosophila embryo.

281 The discovery that many OCRs were unique to either forewings or hindwings may be explained 282 by upstream regulators involved in differentiating the identity of these wings. The Hox gene 283 Ultrabithorax $(U b x)$ is expressed only in hindwing tissue and knocking this gene out transforms 
284 hindwings to forewing identity in butterflies and moths (Matsuoka and Monteiro 2019;

285 Tendolkar et al. 2021). Thus, $U b x$ could be involved in regulating the open chromatin profile that

286 is unique to the hindwings. Loker et al., (2021) found that $U b x$ and its cofactors were important

287 in regulating chromatin accessibility in Drosophila not only between the wing and haltere, but

288 also spatially between the proximal and distal regions of the haltere. Their findings revealed a

289 complex relationship between $U b x$ and its cofactors and how regional differences in the

290 expression of these cofactors influence whether $U b x$ acts as a repressor or an activator of

291 chromatin and gene transcription. Their findings also suggest that $U b x$ plays a key role in

292 regulating chromatin dynamics in the T3 appendages which may explain why in Heliconius and

293 Junonia the major signal in chromatin differences between forewings and hindwings was found

294 at $U b x$ itself. $U b x$ and other Hox genes such as Antennapedia may have a similar role in

295 butterflies, where spatial differences in expression of these genes and their collaborating

296 transcription factors play an important role in regulating pattern development (Beh et al. 2016;

297 Matsuoka and Monteiro 2019; Fang et al. 2021; Paul et al. 2021).

\section{Differential enrichment of transcription factor motifs across wings/regions}

300 Given that Hox genes are important in specifying regional identity between forewings and 301 hindwings (Deutsch 2005; Tomoyasu 2017; Paul et al. 2021) we looked for enrichment of motifs

302 for Hox genes or other transcription factors that may be important in shaping morphological 303 differences between the wings and wing regions. In D. plexippus, we found that a motif for the 304 transcription factor Mad (Mothers against Dpp) and the zinc finger protein Crol (Crooked legs)

305 was significantly enriched in the hindwing relative to the forewing. Surprisingly, despite the 306 requirement of $U b x$ for hindwing identity we found no enrichment of Ubx-binding motifs. A 307 ChiP-seq study against Ubx proteins in Drosophila haltere tissue also found no enrichment of 308 Ubx motifs likely due to its low DNA binding specificity. Instead the study reported enrichment 309 in motifs for GAF (GAGA binding factor) and Mad (Agrawal et al. 2011). The authors suggest 310 that Hox proteins cooperate with other transcription factors in addition to their cofactors to 311 achieve specificity. For example, Mad has previously been shown to collaborate with Ubx in the 312 repression of spalt in halteres (Walsh and Carroll 2007). Whether Mad also collaborates with 313 Ubx in butterfly wings is currently unknown. 
315 Loker et al., (2021) also found no enrichment of the monomer Ubx-binding motif

316 (TAAT)(jaspar.genereg.net/) when conducting a ChiP-seq analysis between the whole imaginal

317 discs of the wing and haltere, however this enrichment was found in the haltere pouch when

318 comparing the proximal and distal regions of the haltere, where Ubx binds alone in the haltere

319 pouch. When we compared the distal and proximal hindwing regions, we found no enrichment of

320 Ubx-binding motifs but instead found enrichment for a motif matching both Escargot and GAF-

321 binding which was significantly enriched in the central region of the OCRs. Escargot regulates

322 genes involved in wing development and can also function as a transcriptional repressor (Fuse et

323 al. 1996). Whether Escargot interacts with Ubx however is unknown. GAF is known to regulate

$324 U b x$ and functions as a pioneer factor involved in both activating and repressing transcription

325 (Biggin and Tjian 1988; Chopra et al. 2008; Lomaev et al. 2017). When comparing the proximal

326 with the distal hindwing region, Bab1 (Bric-a-brac) was significantly enriched in the HWP

327 region. Bab1 interacts with a transcriptional regulator TAF3 (a component of the TFIID

328 complex) which also binds to GAF - a potential cofactor of Ubx (Chopra et al., 2008; Agrawal et

329 al., 2011). Future work could explore whether the different transcription factors enriched in each

330 hindwing region are interacting with Ubx to differentially specify the wing color patterns.

332 In the forewing, motifs for the transcription factors, Jigr1 and Bab1 were significantly enriched 333 relative to the hindwing. Not much is known about the function of Jigr1. In a study examining 334 transcription factor cooperative binding, Kazemian et al., (2013) found that GAF and Jigr1 had 335 the largest number of binding partners which could suggest that Jigr1 has a similar function to 336 GAF in chromatin remodeling. Overall the motif analyses for all wings/wing regions suggests 337 that the enriched motifs in the OCRs include binding sites for transcription factors potentially 338 involved in chromatin remodeling.

340 GC content of OCRs differs between wings and wing regions

341 One of the most striking observations from this analysis was that the different wings/regions were 342 differentiated by the GC content of the transcription factor binding motifs. For both D. plexippus 343 (FW versus HW) and B. anynana (FWD versus HWD), the forewings were characterized by AT344 rich motifs whereas the hindwings were characterized by GC-rich motifs. We also found that the 345 distal and proximal regions of the hindwing differed in the GC content of their motifs. For $D$. 
plexippus, the proximal hindwing region was characterized by AT-rich motifs in contrast to the distal hindwing region which was GC-rich, whereas the opposite trend was found for B. anynana. This difference in GC content of the motifs was explained by the GC content of the OCRs which appeared unrelated to their length. Distal hindwing OCRs had a higher GC content than the overall D. plexippus genome, however this was not due to their length as forewing OCRs were on average

351 longer.

Several studies have shown that GC content is important in determining transcription factor binding. Transcription factors generally exhibit a specific preference not only for their binding motif but also for the sequence properties of the flanking regions (Dror et al. 2015). Some transcription factors prefer to bind at homotypic sites where the flanking regions have a similar GC content to the primary motif, however others prefer contrasting GC content in the 5' and 3' flanking regions (Dror et al. 2015; Dror et al. 2016; Yella et al. 2018). The GC content influences not only the propeller twist of the DNA, and its flexibility or rigidity but also the binding affinity of transcription factors which directly affects gene expression (Dror et al. 2016; Yella et al. 2018). Certain transcription families also show a preference for the GC content and structural features of the DNA. Zinc-finger transcription factors such as GAF, Crol, and Escargot show a preference for binding GC-rich regions whereas homeodomain containing transcription factors prefer AT-rich regions with a low propeller twist (Dror et al., 2016; Yella et al., 2018).

These differences in GC content in open chromatin between the wings and wing regions may represent a form of regulatory control determining which transcription factor complexes are able to bind. In the hindwing for example, these differences could limit the set of transcription factors that interact with Ubx to specify regional identity. As shown by Loker et al., (2021), the cofactors interacting with Ubx varied spatially across the haltere disc activating expression in certain regions yet repressing it in others. Very few ChiP-seq studies have been conducted in Lepidoptera (Burg

372 et al., 2019; Lewis et al., 2016; Lewis et al., 2019b) thus more studies are required to identify spatial patterns of transcription factors binding to open chromatin in wing tissue. 


\section{Sequence conservation of OCRs does not correspond to functional conservation}

378 Although D. plexippus and B. anynana have very different wing patterns, we looked at the

379 chromatin profile around six patterning genes to see whether OCRs that show $>50 \%$ sequence

380 similarity were open in the same wing or wing regions which could imply functional

381 conservation. Overall, we found that many of the conserved OCRs were open in different

382 wings/regions in each species. However, for spalt, $d p p$ and $E c R$, the chromatin is open at the

383 TSS across the entire hindwing in both species. Interestingly, Lewis et al., (2016) found that

384 OCRs at the TSS were more conserved than distal OCRs across lepidopterans. Open chromatin

385 at the TSS could represent expression of these genes. In B. anynana, Spalt proteins and $d p p$

386 mRNA transcripts are present in the marginal eyespots during this developmental period ( $\sim 24$

387 hrs pp) (Banerjee et al. 2021), however we do not have any expression data for the pupal stages

388 of EcR in B. anynana or for any genes in D. plexippus.

390 It is also difficult to know precisely which genes many of these OCRs regulate. We have

391 previously used CRISPR to disrupt one of these conserved OCRs in an intron of $D l l$ in $B$.

392 anynana which led to loss of eyespots and disruption of other traits (Murugesan et al. 2022)

393 mirroring our earlier work knocking out the $D l l$ gene in this butterfly (Connahs et al., 2019). This

394 OCR was open in the distal forewing in B. anynana yet it was open in the proximal hindwing in

395 D. plexippus. Future work characterizing conserved OCRs open either in the same or different

396 wing regions could provide insight into the evolution of their function. As our data represents a

397 single time point, we also do not know whether these conserved OCRs share a similar temporal

398 pattern of accessibility. Lewis et al., (2016) found that OCRs active across multiple

399 developmental stages in butterflies showed increased sequence conservation. Thus, a temporal

400 analysis or functional disruption would help to identify those conserved OCRs that are associated

401 with a specific wing/wing region in both butterfly species which may suggest functional

402 conservation. Taken together our results support previous studies suggesting that sequence

403 conservation does not always imply functional conservation (Nelson and Wardle 2013).

\section{Limitations of the study}

406 The main limitation of this study is that we only had one replicate for each library and thus it is 407 possible that the variation we observed between the different wing regions was due to spurious 
results. However, we think this is unlikely as we observed a similar trend in both butterflies. In both species, we observed variation in the chromatin profile between the different wing regions, and we also observed that OCRs in forewing tissue were AT rich whereas the OCRs in

411 hindwings were GC rich, suggesting that these observations may be a common feature in

412 butterfly wings, however further work is required to validate these findings. Finally, we have

413 confirmed that one of the OCRs identified from this FAIRE-seq data is indeed functional and

414 that knocking it out using CRISPR, leads to phenotypes that would be expected from the

415 disruption of a cis regulatory element that regulates Distal-less (Murugesan et al. 2022).

\section{Conclusions}

418 Here, we have shown that the open chromatin profile varies between different wings and wing 419 regions in two butterfly species revealing how the chromatin landscape can change spatially even 420 within the same tissue. We did not however find a clear association between the chromatin 421 profile and the wing color patterns as demonstrated with the shared OCRs between the distal 422 forewing and proximal hindwing of B. anynana. Our results and those of others highlight the 423 importance of understanding not only the chromatin profile but also the transcription factors that 424 are bound to those OCRs. Although the same OCRs may be open in different wing regions, the 425 binding of transcription factors functioning as activators of nearby genes in one context and as a 426 repressors in another, even in the same tissue, may explain spatial variation in color patterns. 427 Further work is required to understand the role of Hox genes and their cofactors, as well as of 428 other pioneer transcription factors, in generating OCRs, and how structural features of DNA may 429 influence the binding preferences of these and other factors to regulate morphological variation.

Acknowledgements: We thank Christine Merlin, at Texas A\&M University, for sending us

432 Monarch eggs. This work was supported by National Research Foundation (NRF), Singapore

433 under its Investigatorship programme (award NRF-NRFI05-2019-0006).

435 Author contributions: AM and MDG designed the experiment, MDG prepared the samples for 436 FAIRE-seq, HC analyzed the data and wrote the manuscript. AM edited the manuscript. 


\section{Methods}

441 Wild-type B. anynana were maintained in lab populations and reared at $27^{\circ} \mathrm{C}$ and $60 \%$ humidity

442 inside a climate room with 12:12 h light:dark cycle. D. plexippus eggs were obtained from

443 Christine Merlin, at Texas A\&M University, and were reared in the same climate chambers.

444 Bicyclus larvae were supplied with young corn leaves and Danaus with tropical milkweed plants 445 until pupation.

447 Wings from both species were dissected at $22-26$ hours post-pupation. FAIRE-enriched 448 libraries for D. plexippus included 3 libraries, one prepared from whole forewings, and two from 449 partial hindwings (proximal and distal regions). For the control input library (non-enriched), two 450 whole forewings and two whole hindwings were pooled (Fig.1). For B. anynana, three FAIRE451 enriched libraries were prepared including a forewing distal library which had its own control 452 using distal forewing tissue only, and two from partial hindwings (proximal and distal regions) 453 with control inputs of two whole hindwing tissues. All FAIRE-enriched libraries were prepared 454 from 7-8 pooled wing tissues. For this experiment we only collected one replicate per library.

456 Libraries were prepared by Genotypic Technology (India), as paired-end reads (75*2) and 457 sequenced using Illumina NextSeq. Quality checking of the raw reads was performed using 458 FASTQC v0.11.3 and reads which had a phred score>30 were retained for downstream analyses. 459 The reads were aligned to reference scaffolds or genomes for each species (BACs for B. anynana 460 and the whole genome for D. plexippus) with BWA (0.7.13) using the following parameters $-\mathrm{k}$ 461 INT, -w INT, -A INT, -B INT, -O INT, -E INT, -L INT, -U INT. The SAM files were converted 462 to BAM files using SAMtools-0.1.7a and the resulting BAM files were converted to sorted BAM 463 followed by removal of PCR duplicates. The final BAM files were then converted to BEDgraph 464 files using BEDtools-2.14.3. Peaks were called with the MACS2 software using the aligned 465 enriched and input (control) files with the qvalue (minimum FDR) cutoff to call significant 466 peaks. Fold-enrichment and log likelihood scores were calculated using the command bdgcmp 467 script on the enriched and input BEDgraph files. The bdgcmp command also removes noise from 468 the enriched sample relative to the control. The BEDgraph files were converted BigWig files using bdg2bw for visualization in Integrative Genome Viewer (IGV) to identify the peaks 
470 (Thorvaldsdóttir et al. 2013).The BigWig files underlying this article are available in the Dryad

471 Digital Repository which can be found at doi:10.5061/dryad.rv15dv492. The data showing all

472 the peak calls from MACS2 can be found in the supplementary excel files S1-6.

474 Identifying conserved non-coding regions

475 To identify conserved non-coding regions between $B$. anynana and D. plexippus, we used

476 published BAC (Bacterial Artificial Chromosome) sequences from B. anynana around six genes

477 known to be involved in eyespot pattern development (Distal-less, EcR, spalt, dpp, wingless and

478 engrailed), the patterns found in the distal portions of wings (Conceição et al., 2011). The $B$.

479 anynana BACs were approximately $200 \mathrm{~kb}$ so to extract the same regions in D. plexippus we

480 used Ensemble (ensembl.org) to identify the correct genomic location and export 100kb up and

481 downstream of each gene. The genome region was exported along with the annotation files in

482 vista format for use in mVista (Frazer et al. 2004). mVista was used to identify conserved

483 regions between B. anynana and D. plexippus using the Shuffle-Lagan algorithm, which can

484 identify rearrangements, duplications and transposition events and improve alignment of distant

485 homologues (Brudno et al. 2003). Softmasking was used on B. anynana sequences and

486 sequences were reverse-complemented where appropriate. Conservation parameters were

487 changed to a minimum of $50 \%$ identity. The conserved sequences obtained from $\mathrm{mVista}$ along

488 with the FAIRE-seq regions were annotated in geneious version 9.1 (Kearse et al. 2012) to

489 identify overlaps between the conserved regions and the FAIRE-seq peaks.

\section{Motif enrichment and GC content analysis}

492 MEME-chip was used to examine motif enrichment in the regions of open chromatin (Ma et al.

493 2014). MEME-chip is typically used to examine motif enrichment in 100bp peak regions in chip-

494 seq data, however it can also be used locally for analysis of open chromatin by modifying the -

495 ccut parameter from 100 to 0 . This ensures that the whole region of open chromatin is examined

496 rather than just the central $100 \mathrm{bp}$. Regions of open chromatin were extracted from each butterfly

497 genome using bedtools. The databases used for MEME-chip were dmmpmm2009.meme,

498 fly_factor_survey.meme, flyreg.v2.meme and /idmmpmm2009.meme (see S8 for full command

499 line used). The output from MEME-chip produces a motif alignment file which was used to

500 extract the motifs for GC content analysis. Prior to the analysis, duplicate motifs were removed, 
and the motif file was converted to a fasta file. GC content was examined using a python script (see S8 for the command line used). To examine shared regions of open chromatin between different wings and wing regions, bed files were created for each wing group comparison and the command line program Bedops (Neph et al. 2012) was used to generate peak overlap data with a minimum overlap set at $50 \mathrm{bp}$ (see S8 for the command line used). All statistical analyses were performed in Rstudio (RStudio 2020).

\section{Literature cited}

Agrawal P, Habib F, Yelagandula R, Shashidhara LS. 2011. Genome-level identification of targets of Hox protein Ultrabithorax in Drosophila: Novel mechanisms for target selection. Sci. Rep. 1:1-10.

Banerjee T Das, Monteiro A. 2020. Molecular mechanisms underlying simplification of venation patterns in holometabolous insects. Dev. dev.196394.

Banerjee T Das, Ramos D, Monteiro A. 2020. Expression of Multiple engrailed Family Genes in Eyespots of Bicyclus anynana Butterflies Does Not Implicate the Duplication Events in the Evolution of This Morphological Novelty. Front. Ecol. Evol. 8:1-12.

Banerjee T Das, Shan SK, Monteiro A. 2021. optix Is Involved in Eyespot Development Via a Possible Positional Information Mechanism. bioRxiv [Internet]:2021.05.22.445259. Available from: http://biorxiv.org/content/early/2021/05/23/2021.05.22.445259.abstract

Beh CY, El-Sharnouby S, Chatzipli A, Russell S, Choo SW, White R. 2016. Roles of cofactors and chromatin accessibility in Hox protein target specificity. Epigenetics Chromatin [Internet] 9:1. Available from: http://www.pubmedcentral.nih.gov/articlerender.fcgi?artid=4705621\&tool=pmcentrez\&ren dertype $=$ abstract

Biggin MD, Tjian R. 1988. Transcription factors that activate the Ultrabithorax promoter in developmentally staged extracts. Cell 53:699-711.

Bozek M, Cortini R, Storti AE, Unnerstall U, Gaul U, Gompel N. 2019. ATAC-seq reveals regional differences in enhancer accessibility during the establishment of spatial coordinates in the Drosophila blastoderm. Genome Res. 29:771-783.

Brudno M, Malde S, Poliakov A, Do CB, Couronne O, Dubchak I, Batzoglou S. 2003. Glocal 
alignment: Finding rearrangements during alignment. Bioinformatics 19.

Burg KRL Van Der, Lewis JJ, Martin A, Nijhout HF, Danko CG, Reed RD, Burg KRL Van Der, Spineless and EcR in the Highly Dynamic Chromatin Landscape of Butterfly Wing Highly Dynamic Chromatin Landscape of Butter. CellReports [Internet] 27:1027-1038.e3. Available from: https://doi.org/10.1016/j.celrep.2019.03.092

Chopra VS, Srinivasan A, Kumar RP, Mishra K, Basquin D, Docquier M, Seum C, Pauli D,

ConceiçãoInê IC, Long AD, Gruber JD, Beldade P. 2011. Genomic sequence around butterfly wing development genes: Annotation and comparative analysis. PLoS One 6.

Connahs H, Tlili S, van Creij J, Loo TYJ, Banerjee T Das, Saunders TE, Monteiro A. 2019. Activation of butterfly eyespots by Distal-less is consistent with a reaction-diffusion process. Development 146:dev169367.

Deutsch J. 2005. Hox and wings. BioEssays 27:673-675.

Dror I, Golan T, Levy C, Rohs R, Mandel-Gutfreund Y. 2015. A widespread role of the motif environment in transcription factor binding across diverse protein families. Genome Res. 25:1268-1280.

Dror I, Rohs R, Mandel-Gutfreund Y. 2016. How motif environment influences transcription factor search dynamics: Finding a needle in a haystack. BioEssays 38:605-612.

Fang C, Xin Y, Sun T, Monteiro A, Ye Z, Dai F, Lu C, Tong X. 2021. The Hox gene

Ferguson LC, Jiggins CD. 2009. Shared and divergent expression domains on mimetic Heliconius wings. Evol. Dev. [Internet] 11:498-512. Available from: http://www.ncbi.nlm.nih.gov/pubmed/19754707 Antennapedia regulates wing development through 20-hydroxyecdysone in insect. bioRxiv [Internet]:2021.05.26.445904. Available from: http://biorxiv.org/content/early/2021/05/27/2021.05.26.445904.abstract

Ferguson LC, Maroja L, Jiggins CD. 2011. Convergent, modular expression of ebony and tan in the mimetic wing patterns of Heliconius butterflies. Dev. Genes Evol. [Internet] 221:297308. Available from: http://www.ncbi.nlm.nih.gov/pubmed/22139062 
563 Frazer KA, Pachter L, Poliakov A, Rubin EM, Dubchak I. 2004. VISTA: Computational tools for comparative genomics. Nucleic Acids Res. 32:273-279.

Fuse N, Hirose S, Hayashi S. 1996. Determination of wing cell fate by the escargot and snail genes in Drosophila. Development 122:1059-1067.

Hanly JJ, Wallbank RWR, McMillan WO, Jiggins CD. 2019. Conservation and flexibility in the gene regulatory landscape of heliconiine butterfly wings. Evodevo [Internet] 10:1-14. Available from: https://doi.org/10.1186/s13227-019-0127-4

Kazemian M, Pham H, Wolfe SA, Brodsky MH, Sinha S. 2013. Widespread evidence of cooperative DNA binding by transcription factors in Drosophila development. Nucleic Acids Res. 41:8237-8252.

Kearse M, Moir R, Wilson A, Stones-Havas S, Cheung M, Sturrock S, Buxton S, Cooper A, Markowitz S, Duran C, et al. 2012. Geneious Basic: An integrated and extendable desktop software platform for the organization and analysis of sequence data. Bioinformatics 28:1647-1649.

Kunte K, Zhang W, Tenger-Trolander a, Palmer DH, Martin a, Reed RD, Mullen SP, Kronforst MR. 2014. Doublesex Is a Mimicry Supergene. Nature [Internet] 507:229-232. Available from: http://www.ncbi.nlm.nih.gov/pubmed/24598547

Lewis JJ, Geltman RC, Pollak PC, Rondem KE, Belleghem SM Van. 2019. Parallel evolution of ancient, pleiotropic enhancers underlies butterfly wing pattern mimicry. PNAS.

Lewis JJ, Geltman RC, Pollak PC, Rondem KE, Van Belleghem SM, Hubisz MJ, Munn PR, Zhang L, Benson C, Mazo-Vargas A, et al. 2019. Parallel evolution of ancient, pleiotropic enhancers underlies butterfly wing pattern mimicry. Proc. Natl. Acad. Sci. U. S. A.

Lewis JJ, Reed RD. 2018a. Genome-Wide Regulatory Adaptation Shapes Population-Level Genomic Landscapes in Heliconius. :1-15.

Lewis JJ, Reed RD. 2018b. Genome-wide regulatory adaptation shapes population-level genomic landscapes in Heliconius. Mol. Biol. Evol. [Internet]:1-15. Available from: https://academic.oup.com/mbe/advance-article/doi/10.1093/molbev/msy209/5191221 
Loker R, Sanner JE, Mann RS. 2021. Cell-type-specific Hox regulatory strategies orchestrate tissue identity. Curr. Biol. [Internet] 31:4246-4255.e4. Available from: https://doi.org/10.1016/j.cub.2021.07.030

Lomaev D, Mikhailova A, Erokhin M, Shaposhnikov A V., Moresco JJ, Blokhina T, Wolle D, Aoki T, Ryabykh V, Yates JR, et al. 2017. The GAGA factor regulatory network: Identification of GAGA factor associated proteins. PLoS One 12:1-20.

Ma W, Noble WS, Bailey TL. 2014. Motif-based analysis of large nucleotide data sets using MEME-ChIP. Nat. Protoc. 9:1428-1450.

Martin A, Reed RD. 2010. Wingless and aristaless2 define a developmental ground plan for moth and butterfly wing pattern evolution. Mol. Biol. Evol. [Internet] 27:2864-2878. Available from: http://www.ncbi.nlm.nih.gov/pubmed/20624848

Matsuoka Y, Monteiro A. 2018. Melanin Pathway Genes Regulate Color and Morphology of Butterfly Wing Scales. Cell Rep. 24:56-65.

Matsuoka Y, Monteiro A. 2019. Hox genes are essential for the development of novel serial homologous eyespots on the wings of Bicyclus anynana butterflies.

Matsuoka Y, Monteiro A. 2021. Hox genes are essential for the development of eyespots in Bicyclus anynana butterflies. Genetics 217.

Mazo-vargas A, Concha C, Livraghi L, Massardo D, Wallbank RWR, Zhang L. 2017. Macroevolutionary shifts of WntA function potentiate butterfly wing-pattern diversity. PNAS.

McKay DJ, Lieb JD. 2014. A common set of DNA regulatory elements shapes Drosophila appendages. Dev Cell 27.

Monteiro A, Tong X, Bear A, Liew SF, Bhardwaj S, Wasik BR, Dinwiddie A, Bastianelli C, Cheong WF, Wenk MR, et al. 2015. Differential Expression of Ecdysone Receptor Leads to Variation in Phenotypic Plasticity across Serial Homologs. PLOS Genet. [Internet] 11:e1005529. Available from: http://dx.plos.org/10.1371/journal.pgen.1005529

Murugesan SN, Connahs H, Matsuoka Y, Gupta M Das, Huq M, V G, Monroe S, Deem KD, Werner T, Tomoyasu Y, et al. 2022. Butterfly eyespots evolved via co-option of the antennal gene-regulatory network. Press PNAS.

Nelson AC, Wardle FC. 2013. Conserved non-coding elements and cis regulation: Actions speak louder than words. Dev. 140:1385-1395. 
Neph S, Kuehn MS, Reynolds AP, Haugen E, Thurman RE, Johnson AK, Rynes E, Maurano MT, Vierstra J, Thomas S, et al. 2012. BEDOPS: High-performance genomic feature operations. Bioinformatics 28:1919-1920.

Özsu N, Chan QY, Chen B, Gupta M Das, Monteiro A. 2017. Wingless is a positive regulator of eyespot color patterns in Bicyclus anynana butterflies. Dev. Biol. [Internet] 429:177-185. Available from: http://linkinghub.elsevier.com/retrieve/pii/S001216061730132X

Paul R, Giraud G, Domsch K, Duffraisse M, Marmigère F, Khan S, Vanderperre S, Lohmann I, Stoks R, Shashidhara LS, et al. 2021. Hox dosage contributes to flight appendage morphology in Drosophila. Nat. Commun. [Internet] 12. Available from: http://dx.doi.org/10.1038/s41467-021-23293-8

Prakash A, Monteiro A. 2018. Apterous a specifies dorsal wing patterns and sexual traits in butterflies. Proc. R. Soc. B Biol. Sci. 285.

Reed RD, Papa R, Martin A, Hines HM, Counterman BA, Pardo-Diaz C, Jiggins CD, Convergent Evolution of Butterfly Wing Pattern Mimicry. Science [Internet] 333:11371141. Available from: http://www.ncbi.nlm.nih.gov/pubmed/21778360

RStudio. 2020. RStudio: Integrated Development for R. RStudio,. PBC, Boston, MAe.

Shlyueva D, Stampfel G, Stark A. 2014. Transcriptional enhancers: from properties to genomewide predictions. Nat. Rev. Genet. [Internet] 15:272-286. Available from: http://www.ncbi.nlm.nih.gov/pubmed/24614317

Walsh CM, Carroll SB. 2007. Collaboration between Smads and a Hox protein in target gene repression. Development 134:3585-3592.

654 Weatherbee SD, Nijhout HF, Grunert LW, Halder G, Galant R, Selegue J, Carroll S. 1999. 
Biol. 9:109-115.

657 Yella VR, Bhimsaria D, Ghoshdastidar D, Rodríguez-Martínez JA, Ansari AZ, Bansal M. 2018.

658 Flexibility and structure of flanking DNA impact transcription factor affinity for its core

659 motif. Nucleic Acids Res. 46:11883-11897.

660

661

662

663

664

665

666

667

668

669

670

671

672

673

674

675

676

677

678

679

680

681

682

683

684

685

686 


\section{$687 \quad$ Figures}

688

\section{Bicyclus anynana}

\section{Danaus plexippus}
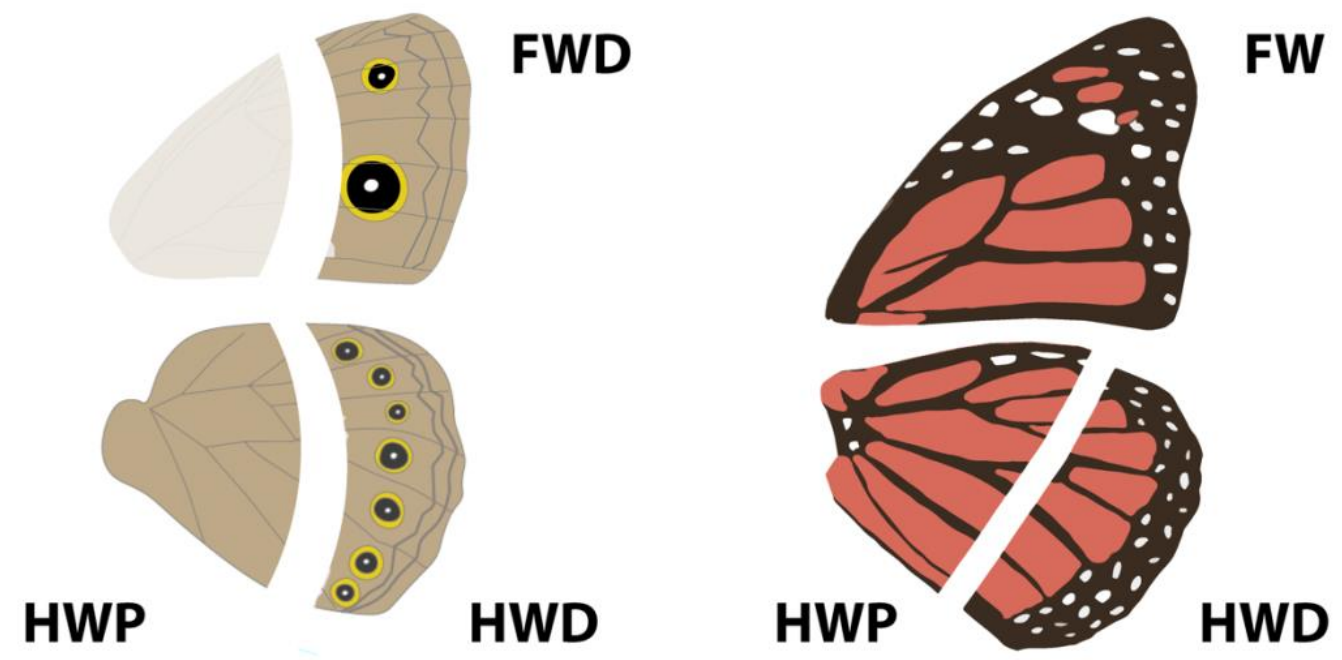

689
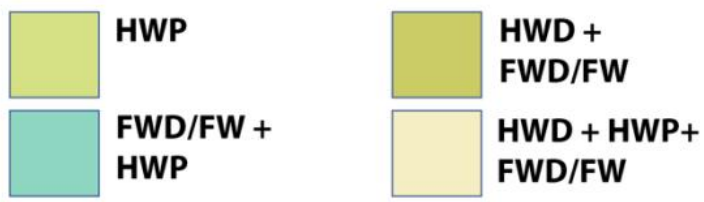

FWD/FW

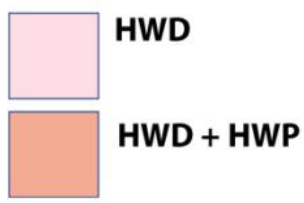

690

691 Fig 1. Wing dissections for FAIRE-seq. For B. anynana, libraries were prepared from the distal

692 forewing (FWD), distal hindwing (HWD) and proximal hindwing (HWP). For D. plexippus,

693 libraries were prepared for the entire forewing (FW), distal hindwing (HWD) and proximal

694 hindwing (HWP). The color key below shows the color scheme that was used to code the

695 significant peaks and those which are overlapping for the FAIRE-seq data (Figs 2-3, S1). 

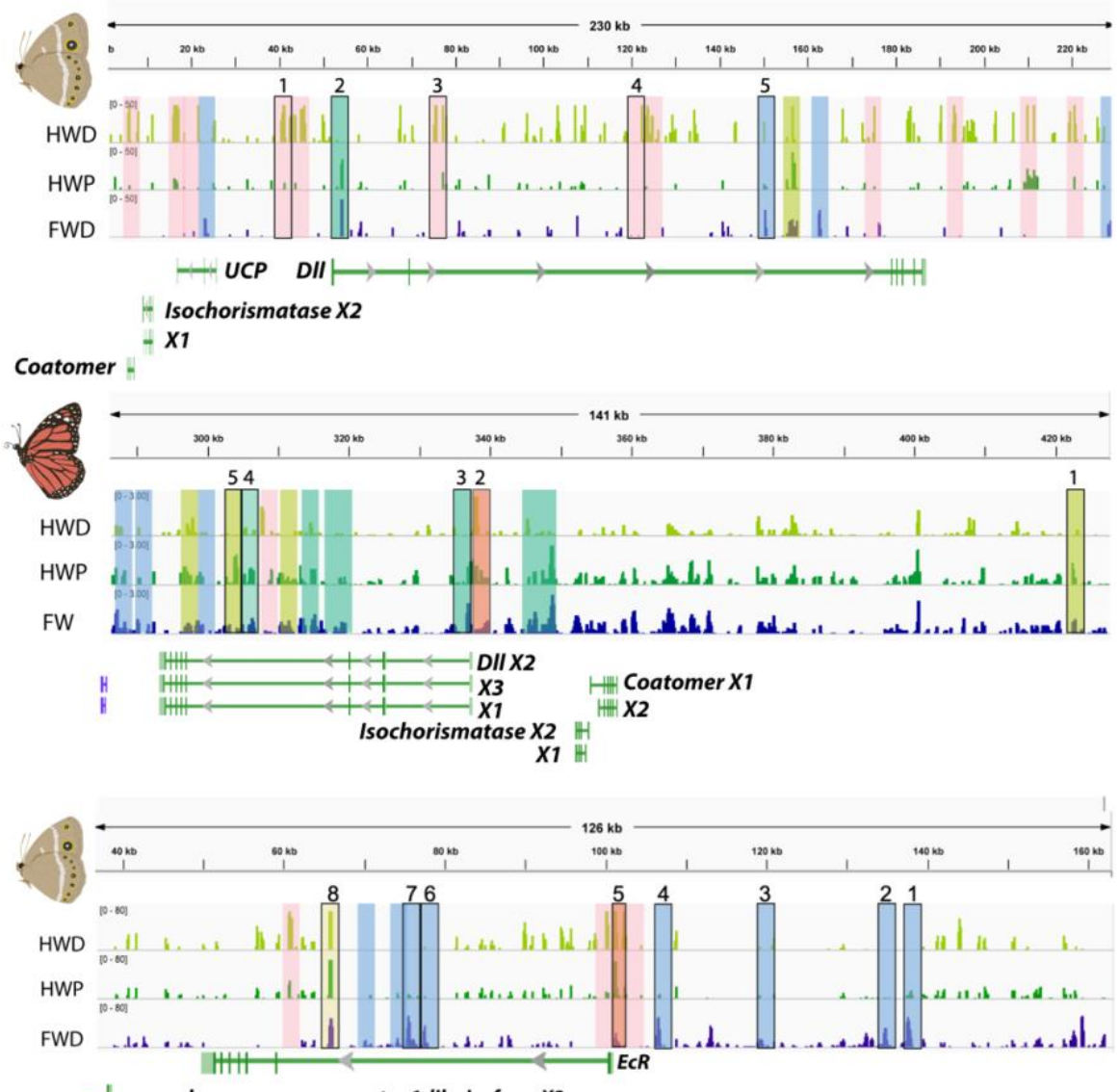

- macrophage mannose receptor 1-like isoform X2 $-x 1$

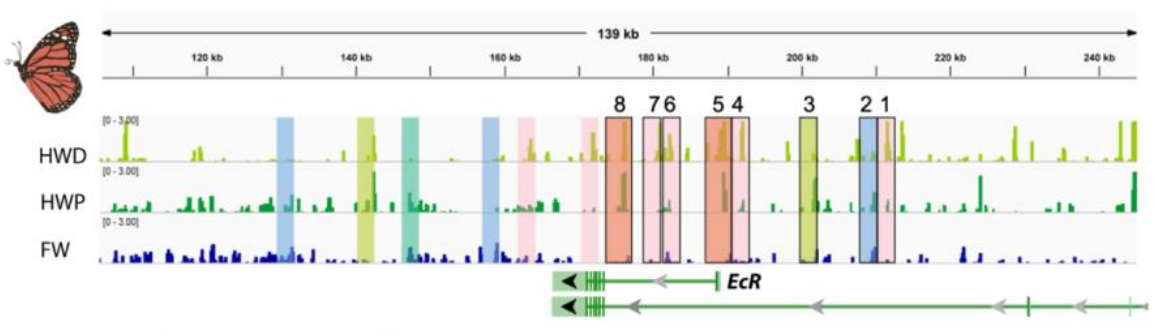

699 Fig 2. FAIRE-seq peaks for B. anynana and D. plexippus around Dll (top panel) and EcR (bottom panel). Significant peaks are colored as follows: Pink - HWD, Green - HWP, Blue -

701 FWD/FW, Orange - overlaps between HWD and HWP, Mint green - overlaps between HWP

702 and FWD/FW, Yellow - overlaps between all 3 wing regions, HWD+HWP+FWD/FW.

703 Conserved peaks identified using mVista (minimum 50\% conservation) are represented by

704 shared numbers. 

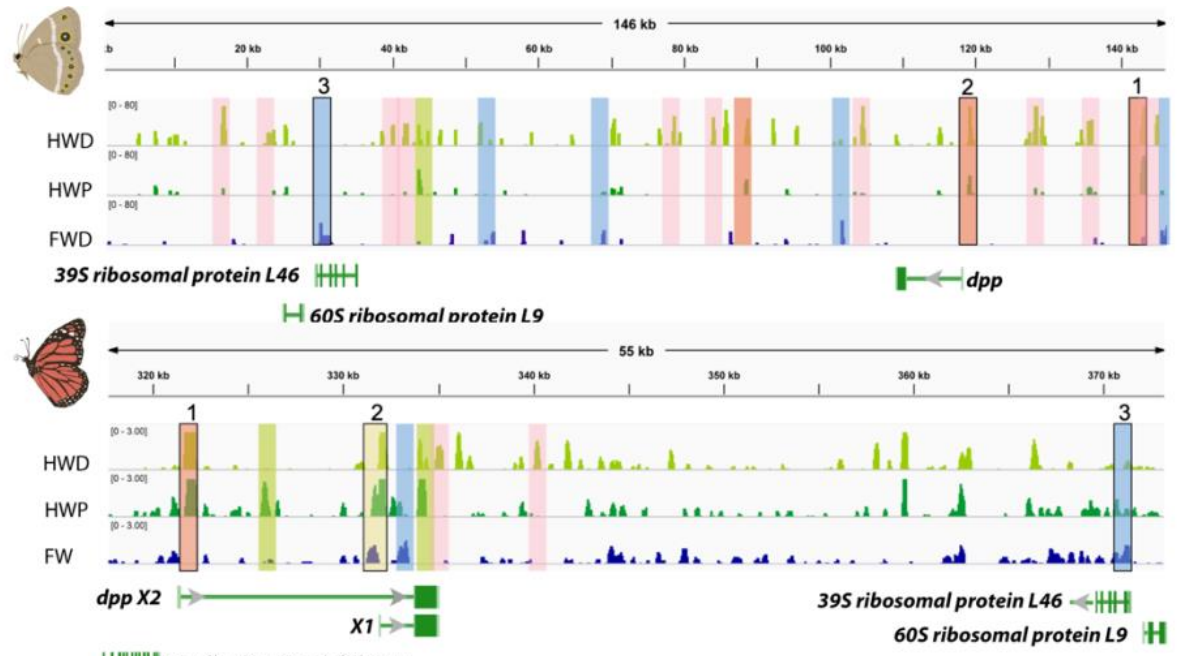

HWWmH T-cell activation inhibitor

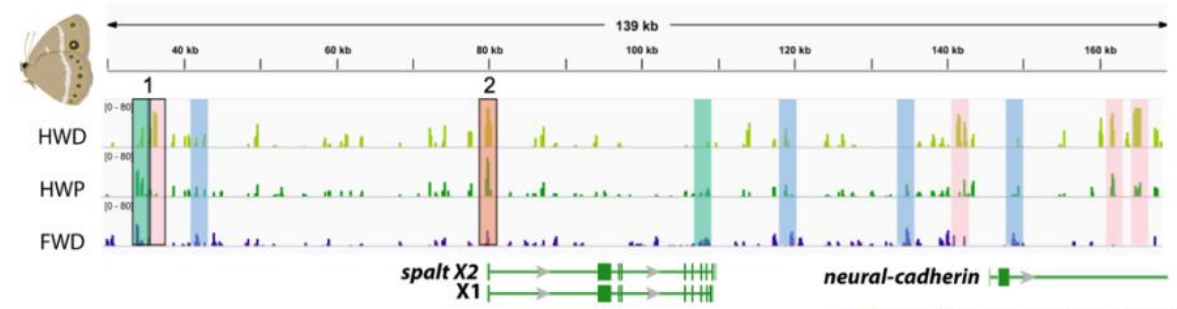

IHWWIH geranylgeranyl transferase HWH 265 proteasome non-ATPase

modifier of mdg4-like $\mathrm{H}$

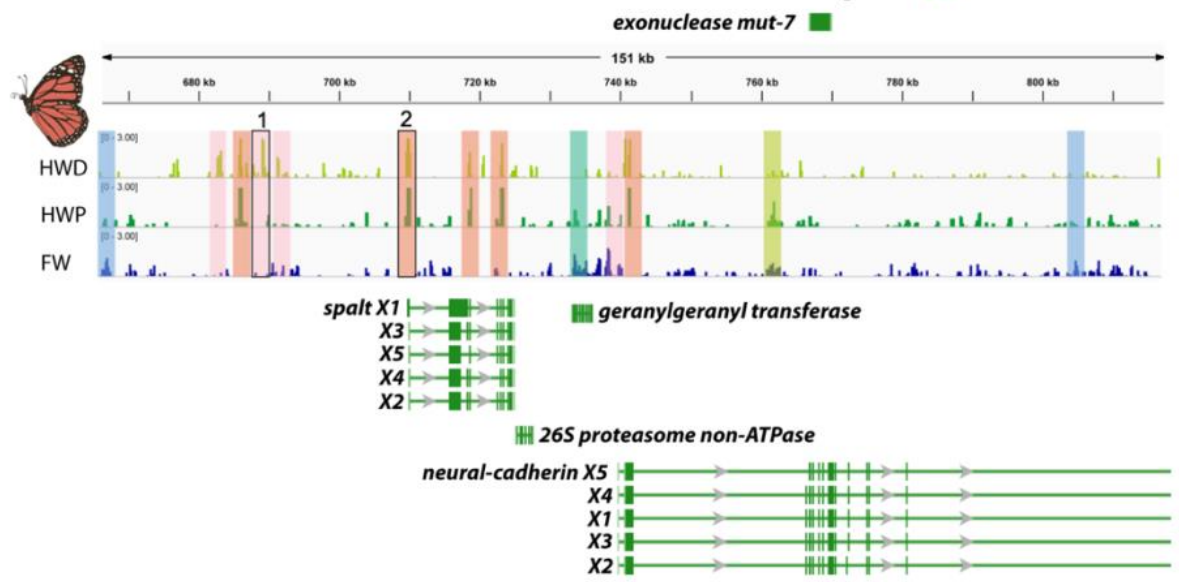

transcription factor GAGA H

exonuclease mut-7

Fig 3. FAIRE-seq peaks for B. anynana and D. plexippus around $d p p$ (top panel) and spalt

(bottom panel). Significant peaks are colored as follows: Pink - HWD, Green - HWP, Blue -

708 FWD/FW, Orange - overlaps between HWD and HWP, Mint green - overlaps between HWP

709 and FWD/FW, Yellow - overlaps between all 3 wing regions, HWD+HWP+FWD/FW.

710 Conserved peaks identified using mVista (minimum 50\% conservation) are represented by

711 shared numbers. 

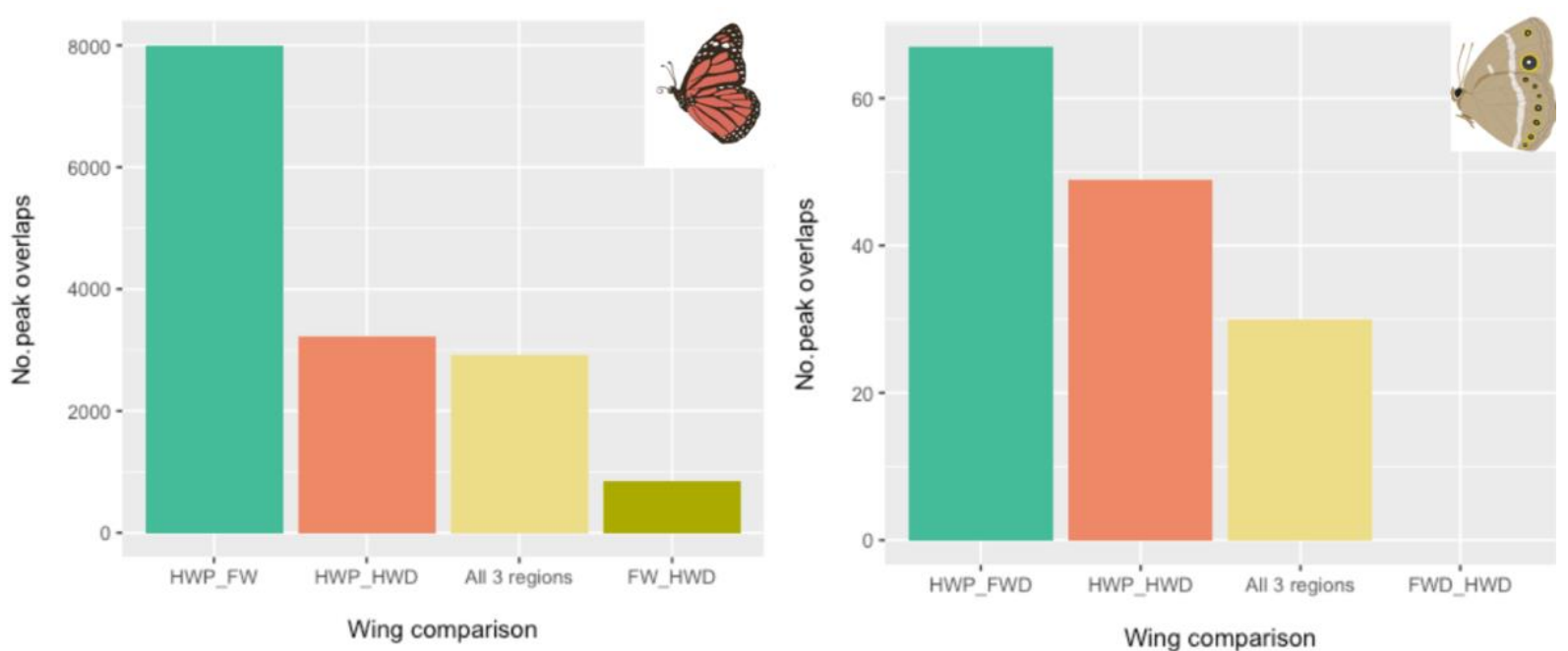

713

714 Fig 4. Number of overlapping OCRs between different wing regions for D. plexippus (based on

715 OCRs extracted from the whole genome) and B. anynana (based on OCRs extracted from 31

716 scaffolds). 
A

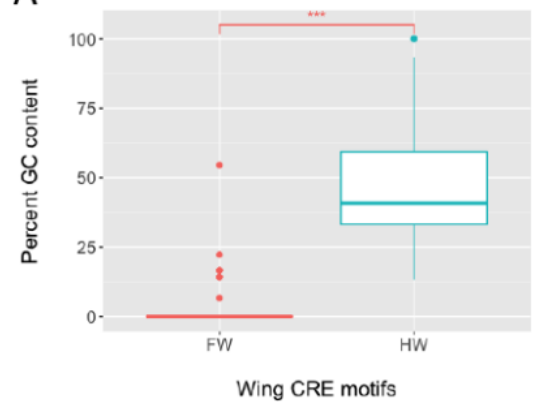

C

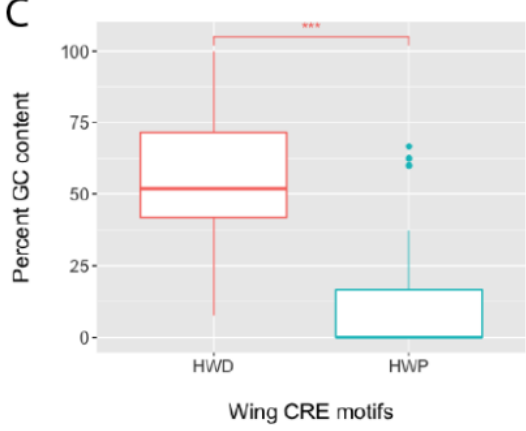

$E$

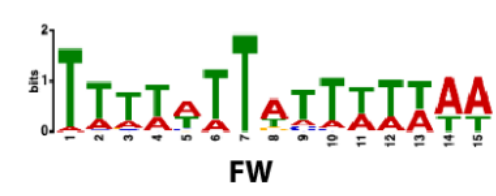

G

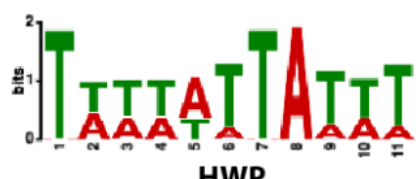

B
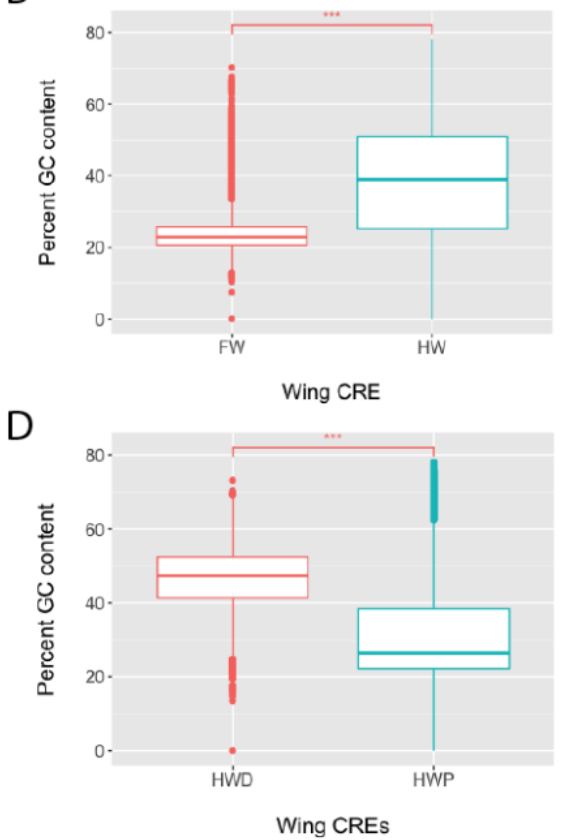

$\mathrm{F}$

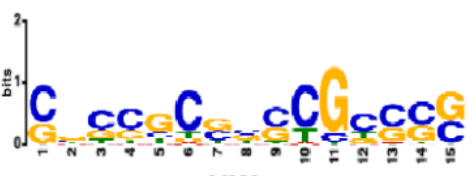

HW

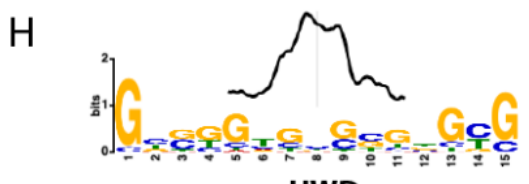

730 Fig 5. GC content analysis for D. plexippus. A) Boxplots showing average percent GC content

731 in motifs in OCRs from forewings (Motifs: FW, $\mathrm{n}=50)$ and hindwings $(\mathrm{HW}, \mathrm{n}=47)$ across the

732 whole genome. B) Average percent GC content in OCRs in forewings (FW, n=21,111) and

733 hindwings (HW, n=20,390) C) Average percent GC content. for motifs for HWD (n=55) and

$734 \operatorname{HWP}(n=37)$ regions and D) in $\operatorname{HWD}(n=8,906)$ and HWP $(n=11,484)$ OCRs across the whole

735 genome. The box represents the interquartile range (IQR), the line represents the median, and

736 the whiskers represent 1.5 times the IQR in the lower and upper quartiles. *** represents

737 P<0.001, E-H) Top hit motifs for MEME using OCRs for the whole genome comparing E) FW

738 versus HW - Jigr1/Bab1, F) HW versus FW - Mad/Crol, G) HWP versus HWD - Bab1, H)

739 HWD versus HWP - Escargot/GAF with the centrimo graph generated by MEME-chip showing

740 that this motif has a centralized position within the OCRs. 